\title{
A Modified Color Display for Computed Tomography
}

\author{
John M. Bramble, Richard Laws, and Michael F. Insana
}

An algorithm for color assignment of computed tomography (CT) scans is presented. The intention is to produce color images similar to illustrations in anatomy atlases. There are three major advantages to the use of this color assignment algorithm. A single color image may replace the multiple monochrome density range pictures now used for portraying CT information. Perception of low spatial frequencies is improved, which may improve detection of soft tissue tumors. Finally, there are economic advantages for using color displays and printed color copies.

( 1988 by W.B. Saunders Company.

KEY WORDS: Color display, computed tomography, color assignment, radiology display, color hard copy.

C OLOR DISPLAYS of computed tomography (CT) images have advantages over monochrome displays but have not been accepted by radiologists. The primary advantage of a color display is to show a wider range of contrast. Use of color displays permits multiple density ranges (ie, bone, soft tissue, and lung densities) to be presented in one image. Nearly all of the contrast in a CT scan may be displayed in one color window. A second advantage of color display is improved perception of low spatial frequncies ${ }^{1}$ which can increase the detectability of tumors in soft tissues.

Color displays have not been accepted for general use. The reasons for rejection include expense of color film and perceptual difficulties. The ability to gestalt images is hampered by contours that appear with the use of color. In addition, the dazzling array of colors tends to detract from viewing the image.

The economic reasons for avoiding color displays may soon be reversed. Because of engineering demand, the cost of high resolution $(1,024$ pixel square resolution) color monitors is less than monochrome monitors. More importantly, printing technology is advancing to the point that high resolution color printing is available with greater contrast resolution (due to use of colors) than gray scale printers. Such color-printed hard copy would be cheaper than film.

Modifications can be made to color displays to reduce objection of radiologists. The primary goals of such modifications are to (1) maintain the ability to display all information in one image (ie, identify bone, soft tissue, and lung abnormal- ities in one window), (2) reduce the contours that detract from interpreting the image, and (3) limit colors to avoid distraction. To accomplish this objective we developed a technique that combines several previously described methods and applies them in a unique way.

The basic method of the modified color display is assignment of a specific hue and range of intensity to the range of pixel values (a color look-up table). Since the majority of high resolution color monitors have RGB (red-green-blue) inputs, it is necessary to translate intensity and hue into values of red, green, and blue intensities. This is accomplished by the use of an IHS (intensity hue saturation) transform as described by Farrell. ${ }^{2}$

The next step is the selection of intensity and hues for encoding the pixel values. In a CT image we can expect resolution of attenuation values for air, fat, soft tissue, and bone. Problems arise in classifying each pixel into one of the aforementioned because of partial volume artifacts (especially prevalent in the lungs) and beam hardening artifacts (noticeable in variations of pixel attenuation values in peripheral $v$ central portions of the image). Partial volume artifacts are the major source of inaccurate classification based on pixel value.

A hue is selected for each category (air, fat, soft tissue, and bone). Variations in the attenuation within each class are encoded as intensity variations. For example, bone with higher attenuation values (cortical bone) is encoded by a high intensity neutral hue (white). Bone with lower attenuation values (medullary bone) is encoded by a lower intensity neutral hue (gray). Encoding bone with neutral hues takes advantage of improved high spatial frequency achromatic response. ${ }^{1}$ The

From the Department of Diagnostic Radiology, Section of Musculoskeletal Radiology, and the Department of Diagnostic Radiology, Division of Diagnostic Imaging and Radiological Sciences, University of Kansas Medical Center, Kansas City.

Address reprint requests to John $M$. Bramble, MD, Department of Diagnostic Radiology, Section of Musculoskeletal Radiology, The University of Kansas Medical Center, 39th St and Rainbow Blvd, Kansas City, KS.

(C) 1988 by W.B. Saunders Company.

0897-1889/88/0101-0003\$03.00/0 

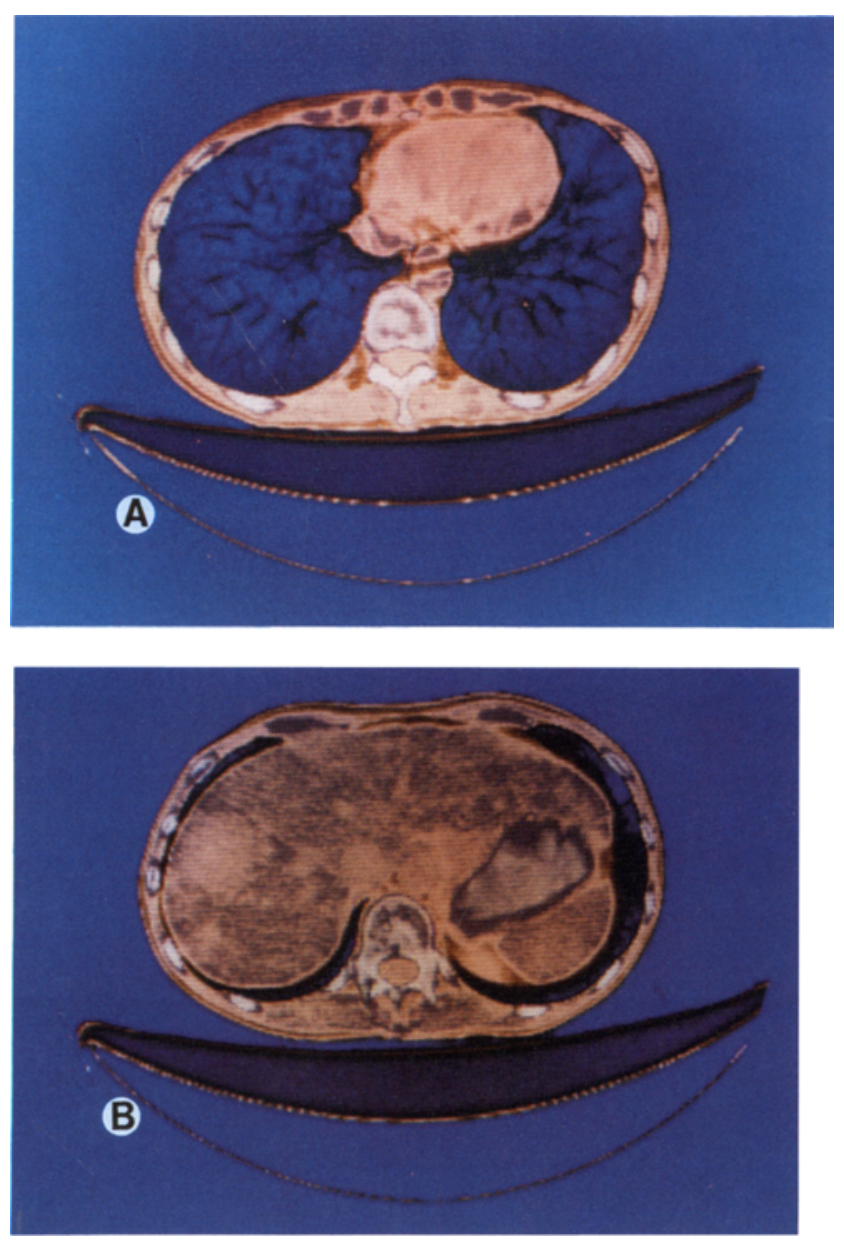

sharper edges of bone are more clearly perceived. Soft tissue with a higher attenuation (liver) is encoded by a higher intensity reddish brown color than lower intensity muscle. Attenuation values for fluids are displayed as dark brown. For fat, the gradient is the same but the hue is yellow. For lungs the classification breaks down because of partial volume effects. A wide range of values is encountered for pulmonary vessels which are encoded by variations in intensity as well as hue (shifting from blue to red).

The resulting images are presented for the reader's appraisal. In our own department, body imaging specialists who routinely use monochrome images have generally expressed that the modified color display is an improvement over prior color displays. However, the body imaging specialists still prefer monochrome displays. The preference for monochrome displays probably reflects long-term habits.

The modified color display has achieved the goals of displaying subtle soft tissue metastases,
Fig 1. (A) CT axial section showing lung markings and blastic metastases (prostate) in vertebral body. (B) CT axial section through liver with same window settings as (A). High tissue contrast in liver shows multiple metastatic lesions. A blastic metastasis is also visible in the vertebrae body. bone metastases, and lung detail on one image (Fig 1). The modified color displayed is clearly easier to gestalt than the standard color displays because of reductions in contouring and brightness. However, radiologists could not be expected to modify viewing habits unless a strong economic incentive is present. In the near future, both video display and printing technology advances will result in color images being cheaper than monochrome images and may provide the economic incentive for re-evaluating the use of color for CT images. Furthermore, clinicians are less ingrained in viewing habits. Color copies of exams might be provided to clinicians. The distribution of printed color copies would reduce the price of the service to provide such copies.

\section{REFERENCES}

1. Pratt WK: Digital Image Processing. New York, Wiley, 1978

2. Farrell EJ: Color display and interactive interpretation of three-dimensional data. IBM J Res Develop 27:356-366, 1983 